\title{
ROTH'S EQUIVALENCE PROBLEM IN UNIT REGULAR RINGS
}

ROBERT E. HARTWIG

\begin{abstract}
It is shown that for matrices over a unit regular ring $\left[\begin{array}{ll}A & C \\ 0 & D\end{array}\right]$ $\sim\left[\begin{array}{ll}A & 0 \\ 0 & D\end{array}\right]$ if and only if there exist solutions $X$ and $Y$ to $A X-Y D=C$, thus providing a partial generalization to Roth's theorem.
\end{abstract}

1. Introduction. One of the most remarkable consistency conditions for the existence of a solution $X$ to the matrix equation

$$
A X-X D=C
$$

was given by Roth [14], who showed that for matrices over a field $\mathscr{F}$, (1) has a solution $X$ if and only if

$$
M=\left[\begin{array}{ll}
A & C \\
0 & D
\end{array}\right] \approx\left[\begin{array}{ll}
A & 0 \\
0 & D
\end{array}\right]=N,
$$

where $\approx$ denotes similarity. He proved that this result was a consequence of the fact that for $\lambda$-matrices over $\mathscr{F}[\lambda]$

$$
A(\lambda) X(\lambda)-Y(\lambda) D(\lambda)=C(\lambda)
$$

has solutions $X(\lambda), Y(\lambda)$ if and only if

$$
\left[\begin{array}{cc}
A(\lambda) & C(\lambda) \\
0 & D(\lambda)
\end{array}\right] \sim\left[\begin{array}{cc}
A(\lambda) & 0 \\
0 & D(\lambda)
\end{array}\right]
$$

where $\sim$ denotes equivalence [6, p. 132]. A different proof for matrices over a field was given by Flanders and Wimmer [4]. The existence of solutions to (1) and (3) immediately implies the desired similarity and equivalence of the matrices in (2) and (4) respectively. Indeed, as shown in [14],

$$
\begin{aligned}
& {\left[\begin{array}{ll}
I & X \\
0 & I
\end{array}\right]\left[\begin{array}{ll}
A & C \\
0 & D
\end{array}\right] \quad\left[\begin{array}{cc}
I & -X \\
0 & I
\end{array}\right]=\left[\begin{array}{ll}
A & 0 \\
0 & D
\end{array}\right],} \\
& {\left[\begin{array}{ll}
I & Y \\
0 & I
\end{array}\right] \quad\left[\begin{array}{ll}
A & C \\
0 & D
\end{array}\right] \quad\left[\begin{array}{cc}
I & -X \\
0 & I
\end{array}\right]=\left[\begin{array}{ll}
A & 0 \\
0 & D
\end{array}\right] .}
\end{aligned}
$$

Received by the editors June 4, 1975.

AMS (MOS) subject classifications (1970). Primary 15A33, 15A09, 16A30.

(6) American Mathematical Society 1976 
We may consequently state Roth's problems as follows:

(i) Roth's similarity problem. For what types of matrices does the similarity of $\left[\begin{array}{ll}A & C \\ 0 & D\end{array}\right]$ and $\left[\begin{array}{ll}A & 0 \\ 0 & D\end{array}\right]$ ensure the existence of a solution $X$ to $A X-X D=C$ ?

(ii) Roth's equivalence problem. For what types of matrices does the equivalence of $\left[\begin{array}{cc}A & C \\ 0 & D\end{array}\right]$ and $\left[\begin{array}{ll}A & 0 \\ 0 & D\end{array}\right]$ ensure the existence of solutions $X$ and $Y$ to $A X-Y D=C$ ?

The purpose of this note is to show that for matrices over a unit regular ring and, in particular, for skewfields, the answer to Roth's equivalence problem is affirmative. Generalizing a result by Meyer [12], we shall see that for a regular ring this problem is equivalent to the existence of an upper triangular matrix solution to $M X M=M$.

A ring $\Re$ is called regular if for every $a \in \Re$, there exists a solution $a^{-} \in \Re$ to the equation $a x a=a$. The solution $a^{-}$is called an inner or 1 inverse of $a$. Similarly any solution $a^{+} \in \Re$ to the equations $a x a=a, x a x=x$, is called a reflexive or 1-2 inverse of $a$ [1]. A regular ring with unity 1 is called unit regular if every $a \in \Re$ possess a unit (=2 sided invertible) inner inverse $a^{-} \in \mathcal{R}$ [2]. A ring $\Re$ with unity 1 is called finite if $a b=1$ implies $b a=1$ [10]. It was shown by Henriksen [10] that if $R$ is unit regular then so is $\Re_{n \times n}$, the ring of $n \times n$ matrices over $R$, and hence in the case of square matrices we may, in Roth's problems, without loss of generality, work with ring elements. Matrices over unit regular rings behave in many respects like matrices over fields. They are diagonalizable under equivalence, one sided inverses are two sided inverses, and they have invertible inner inverses. For a proof of these results we refer to [10].

We shall denote the ring of $n \times 1$ column vectors over $\Re$ by $\Re^{n}$, isomorphisms by $\cong$, and rank by $\rho(\cdot)$. By $b \sim a$ we shall mean $b=$ paq with $p, q$ invertible, and we define $a^{0}=\{x \in \mathcal{R} ; a x=0\},{ }^{0} a=\{x \in \mathscr{R} ; x a=0\}$.

2. Main results. We begin by observing that "finiteness" of ring $\mathscr{R}$ is certainly necessary for (2) to imply (1). Indeed, if $b a=1 \neq a b$, then

$$
\begin{aligned}
{\left[\begin{array}{cc}
b & 0 \\
1-a b & a
\end{array}\right]\left[\begin{array}{cc}
a & 1-a b \\
0 & b
\end{array}\right] } & =\left[\begin{array}{ll}
1 & 0 \\
0 & 1
\end{array}\right] \\
& =\left[\begin{array}{cc}
a & 1-a b \\
0 & b
\end{array}\right]\left[\begin{array}{cc}
b & 0 \\
1-a b & 0
\end{array}\right]
\end{aligned}
$$

and

$$
\left[\begin{array}{cc}
a & 1-a b \\
0 & b
\end{array}\right]\left[\begin{array}{cc}
a & 1-a b \\
0 & 0
\end{array}\right]\left[\begin{array}{cc}
b & 0 \\
1-a b & a
\end{array}\right]=\left[\begin{array}{ll}
a & 0 \\
0 & 0
\end{array}\right]
$$

showing that

$$
\left[\begin{array}{cc}
a & 1-a b \\
0 & 0
\end{array}\right] \approx\left[\begin{array}{ll}
a & 0 \\
0 & 0
\end{array}\right]
$$


Yet there can be no solution to $a x-0 d=1-a b$ since this would imply $x=b a x=b(1-a b)=0$ and thus $1=a b$, which is impossible. Hence finiteness is at least necessary locally [13].

We are now ready to state our main theorem, extending Roth's equivalence problem to unit regular rings, thereby generalizing the results of [4] and [12].

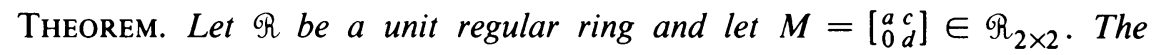
following are equivalent.

(1) $M$ has an inner inverse of the form $\left[\begin{array}{ll}r & s \\ 0 & t\end{array}\right]$.

(2) $a x-y d=c$ has solutions $x, y \in \Re$.

(3) $\left(1-a a^{-}\right) c\left(1-d^{-} d\right)=0$ for all $a^{-}, d^{-}$.

(4) ${ }^{0} a c d^{0}=(0)$.

(5) $M \sim\left[\begin{array}{ll}a & 0 \\ 0 & d\end{array}\right]$.

(6) $\left(1-a a^{+}\right) c\left(1-d^{+} d\right)=0$ for all $a^{+}, d^{+}$.

$$
M^{+}=\left[\begin{array}{cc}
a^{+} & -a^{+} c d^{+} \\
0 & d^{+}
\end{array}\right] \text {is a reflexive inverse for } M
$$

In the cases where $a, c$ and $d$ are $n \times n$ matrices over a skewfield or commutative ring without zero divisors, these are equivalent to

(5a) $\rho(M)=\rho(a)+\rho(d)$.

(5b) $\rho\left[\left(1-a a^{+}\right) c\left(1-d^{+} d\right)\right]=0$.

Proof. (1) $\Rightarrow$ (2). Suppose

$$
\left[\begin{array}{ll}
a & c \\
0 & d
\end{array}\right]\left[\begin{array}{ll}
r & s \\
0 & t
\end{array}\right]\left[\begin{array}{ll}
a & c \\
0 & d
\end{array}\right]=\left[\begin{array}{ll}
a & c \\
0 & d
\end{array}\right]
$$

then ara $=a, d t d=d$ and $a(r c)+(a s+c t) d=c$, showing that we may take $x=r s$ and $y=-(a s+c t)$.

$(2) \Rightarrow(3)$.

$$
\begin{aligned}
a x-y d & =c \Rightarrow 0=\left(1-a a^{-}\right)[a x-y d]\left(1-d^{-} d\right) \\
& =\left(1-a a^{-}\right) c\left(1-d^{-} d\right) .
\end{aligned}
$$

(3) $\Rightarrow(4)$. Clearly

$$
\left(1-a a^{-}\right) c\left(1-d^{-} d\right)=0 \Leftrightarrow \Re\left(1-a a^{-}\right) c\left(1-d^{-} d\right) \Re=(0) .
$$

$(4) \Rightarrow(5)$. This is contained in (6).

$(5) \Rightarrow(6)$. This is the only part of the theorem that requires the unit regularity of $\Re$. We begin by noting that if $a^{+}$is any 1-2 inverse of $a$, then $a a^{+} \Re=a \Re$. Consider now the matrix

$$
M_{1}=\left[\begin{array}{cc}
1 & -\left(1-a a^{+}\right) c d^{+} \\
0 & 1
\end{array}\right]\left[\begin{array}{ll}
a & c \\
0 & d
\end{array}\right]\left[\begin{array}{cc}
1 & -a^{+} c \\
0 & 1
\end{array}\right]=\left[\begin{array}{ll}
a & p \\
0 & d
\end{array}\right],
$$

where $p=\left(1-a a^{+}\right) c\left(1-d^{+} d\right)$. Then $M_{1} \sim N$ and $M_{1}=P N Q$ for suitable 
invertible $P$ and $Q$. We now claim that [7]

$$
M_{1}^{+}=\left[\begin{array}{cc}
a^{+} & 0 \\
\left(1-d^{+} d\right) p^{+}\left(1-a a^{+}\right) & d^{+}
\end{array}\right] \quad \text { and } \quad N^{+}=\left[\begin{array}{cc}
a^{+} & 0 \\
0 & d^{+}
\end{array}\right]
$$

are particular reflexive inverses for $M_{1}$ and $N$ respecitvely. Indeed,

$$
E=M_{1} M_{1}^{+}=\left[\begin{array}{cc}
a a^{+}+p p^{+}\left(1-a a^{+}\right) & 0 \\
0 & d d^{+}
\end{array}\right]=\left[\begin{array}{cc}
e & 0 \\
0 & f
\end{array}\right],
$$

with $e^{2}=e, f^{2}=f$, from which it easily follows that $M M^{+} M=M$, and $M^{+} M M^{+}=M^{+}$since $a^{+} p=0=p d^{+}$. The result for $N^{+}$is trivial. Since $M_{1} \mathcal{R}_{2 \times 2}=M_{1} M_{1}^{+} \Re_{2 \times 2}$ we see that $M_{1}=P N Q$ implies that

$$
M_{1} M_{1}^{+} \Re_{2 \times 2}=M_{1} \Re_{2 \times 2}=P N \Re_{2 \times 2} \cong N \Re_{2 \times 2}=N N^{+} \Re_{2 \times 2},
$$

as right $\Re_{2 \times 2}$ modules. We now apply the following result of [8].

Lemma 1. For any two idempotent matrices $E$ and $F$ over any ring $R$ with identity, the following are equivalent:

(i) $E \Re_{2 \times 2} \cong F \Re_{2 \times 2}$ as right $\Re_{2 \times 2}$-modules,

(ii) $E \Re^{2} \cong F \Re^{2}$ as right $\Re$-modules.

Since $E=M_{1} M_{1}^{+}$and $F=N N^{+}$are idempotent we obtain

$$
\begin{aligned}
{\left[\begin{array}{l}
e \Re \\
f \Re
\end{array}\right]\left[\begin{array}{ll}
e & 0 \\
0 & f
\end{array}\right] \Re^{2} } & =M_{1} M_{1}^{+} \Re^{2} \\
& \cong N N^{+} \Re^{2}=\left[\begin{array}{ll}
g & 0 \\
0 & f
\end{array}\right] \Re^{2}=\left[\begin{array}{l}
g \Re \\
f \Re
\end{array}\right],
\end{aligned}
$$

where $g=a a^{+}, f=d d^{+}$and $e=a a^{+}+p p^{+}\left(1-a a^{+}\right)$.

Because $\Re$ is unit regular we may now use the following cancellation law [9] for external direct sums:

LEMMA 2.

$$
e \mathfrak{R} \oplus f \mathfrak{R} \cong e^{\mathscr{R}} \oplus g \mathfrak{R} \Rightarrow f \mathfrak{R} \cong g^{\Re} \text { as right } \mathfrak{R} \text {-modules. }
$$

Proof. This law is a consequence of the unit regularity of $\Re_{2 \times 2}[10]$ and the fact that [15] is a unit regular $\operatorname{ring} g \Re \cong h \Re, g^{2}=g, h^{2}=h \Rightarrow g \approx h$. Indeed, denoting internal direct sums by $\dot{+}$, and using Lemma 1 , we have

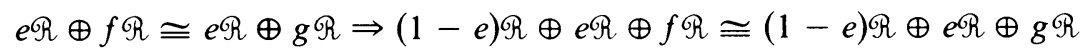

$\Rightarrow((1-e) \Re \dot{+} e \Re) \oplus f \Re \cong((1-e) \Re \dot{+} e \Re) \oplus g \Re$

$\Rightarrow \Re \oplus f \mathfrak{R} \cong \Re \oplus g \Re$

$\Rightarrow\left[\begin{array}{ll}1 & 0 \\ 0 & f\end{array}\right] \Re^{2} \cong\left[\begin{array}{ll}1 & 0 \\ 0 & g\end{array}\right] \Re^{2}$ as $\Re$-modules 


$$
\begin{aligned}
& \Rightarrow\left[\begin{array}{ll}
1 & 0 \\
0 & f
\end{array}\right] \Re_{2 \times 2} \cong\left[\begin{array}{ll}
1 & 0 \\
0 & g
\end{array}\right] \Re_{2 \times 2} \text { as } \Re_{2 \times 2} \text {-modules } \\
& \Rightarrow\left[\begin{array}{cc}
0 & 0 \\
0 & 1-f
\end{array}\right] \Re_{2 \times 2} \cong\left[\begin{array}{cc}
0 & 0 \\
0 & 1-g
\end{array}\right] \Re_{2 \times 2} \quad \text { as } \Re_{2 \times 2} \text {-modules } \\
& \Rightarrow\left[\begin{array}{cc}
0 & 0 \\
0 & 1-f
\end{array}\right] \Re^{2} \cong\left[\begin{array}{cc}
0 & 0 \\
0 & 1-g
\end{array}\right] \Re^{2} \quad \text { as } \Re \text {-modules } \\
& \Rightarrow(1-f) \mathscr{R} \cong(1-g) \Re \Rightarrow f \mathscr{R} \cong g \mathcal{R} .
\end{aligned}
$$

Similar cancellation laws were discussed by Fuchs [5, p. 201], and Ehrlich [3]. Application of the cancellation law (14) of (13) shows that $e^{\mathscr{R}} \cong g \Re$. Now since $a^{+} p=0=\left(1-a a^{+}\right) a$ we see that

$$
\begin{aligned}
a a^{+} \Re+p p^{+}\left(1-a a^{+}\right) \Re & =\left[a a^{+}+p p^{+}\left(1-a a^{+}\right)\right] \Re \\
& =e \mathscr{R} \cong g \Re=a a^{+} \Re=a \Re
\end{aligned}
$$

and also $a a^{+} \Re \oplus p p^{+}\left(1-a a^{+}\right) \Re \cong a \Re$.

Using the cancellation law (14) once more shows that $p p^{+}\left(1-a a^{+}\right) \mathscr{R}$ $\cong(0)$, and hence $p p^{+}\left(1-a a^{+}\right) \mathscr{R}=0$. This in turn implies that $p p^{+}\left(1-a a^{+}\right)$ $=0$ so that $p=p p^{+} p=p p^{+}\left(1-a a^{+}\right) c\left(1-d^{+} d\right)=0$, completing the proof.

(6) $\Rightarrow$ (7). If $\left(1-a a^{+}\right) c\left(1-d^{+} d\right)=0$ then $a x-y d=c$ has solutions $x=a^{+} c\left(1-d^{+} d\right), y=-c d^{+}$or $x=a^{+} c, y=\left(a a^{+}-1\right) c d^{+}$. From (9) and (10) with $p=0$ we see that the matrix

$$
M^{+}=\left[\begin{array}{cc}
1 & -a^{+} c \\
0 & 1
\end{array}\right]\left[\begin{array}{cc}
a^{+} & 0 \\
0 & d^{+}
\end{array}\right]\left[\begin{array}{cc}
1 & -\left(1-a a^{+}\right) c d^{+} \\
0 & 1
\end{array}\right]=\left[\begin{array}{cc}
a^{+} & -a^{+} c d^{+} \\
0 & d^{+}
\end{array}\right]
$$

is a reflexive inverse for $M$.

(7) $\Rightarrow(1)$. Obvious.

For matrices over a skewfield or any commutative ring without zero divisors we may use rank $\rho(\cdot)([11]$, [7]) to give a much shorter proof that (5) implies (6) using (5a)-(5b). Indeed, if $\left[\begin{array}{ll}a & p \\ 0 & d\end{array}\right] \sim\left[\begin{array}{ll}a & 0 \\ 0 & d\end{array}\right]$ then $\rho(M)=\rho(a)+\rho(d)$. But it follows from the rank formula [7, Equation (4.19)],

$$
\rho\left[\begin{array}{ll}
a & c \\
b & d
\end{array}\right]=\rho(a)+\rho\left[\begin{array}{cc}
0 & \left(1-a a^{+}\right) c \\
b\left(1-a^{+} a\right) & d-b a^{+} c
\end{array}\right]
$$

that

$$
\rho(a)+\rho(d)=\rho\left[\begin{array}{ll}
a & 0 \\
0 & d
\end{array}\right]=\rho\left[\begin{array}{ll}
a & p \\
0 & d
\end{array}\right] \rho(a)+\rho\left[\begin{array}{l}
p \\
d
\end{array}\right]=\rho(a)+\rho(p)+\rho(d) .
$$

Hence $\rho(p)=0$ and thus $p=0$ as desired.

We conclude with the following remarks. 
3. Conclusions. We have thus seen that Roth's equivalence theorem holds for matrices over Euclidean domains as well as unit regular rings, both of which are finite rings, elementary divisor rings, and obey the cancellation law (14). It is an open question whether these properties suffice for Roth's equivalence theorem to hold. It also remains an open question whether unit regularity suffices for Roth's similarity problem. The above method does not give any indication since it makes extensive use of idempotent matrices for which equivalence and similarity are synonymous.

Acknowledgement. The author wishes to thank Dr. J. Luh for many stimulating discussions.

\section{REFERENCES}

1. A. Ben Israel and T. N. E. Greville, Generalized inverses theory and applications, Wiley, New York, 1974.

2. G. Ehrlich, Unit-regular rings, Portugal. Math. 27 (1968),209-212. MR 42 \# 1864.

3. - Units and one-sided units in regular rings, Trans. Amer. Math. Soc. 216 (1976), 81-90.

4. $\mathrm{H}$. Flanders and $\mathrm{H}$. Wimmer, Uber die Matrizen gleichungen $A X-X B=C$ and $A X-Y B$ $=C$, Arch. Math. (to appear).

5. L. Fuchs, On a substitution property of modules, Monatsh. Math. 75 (1971), 198-204. MR 45 \#5157.

6. F. R. Gantmacher, The theory of matrices, GITTL, Moscow, 1953; English transl., Vol. 1, Chelsea, New York, 1959. MR 16, 438; 21 \#6372c.

7. R. E. Hartwig, Block generalized inverses, Arch. Rational Mech. Anal. 61 (1976).

8. - Generalized inverses, EP elements and associates (to appear).

9. R. E. Hartwig and J. Luh, A note on finite regular rings, Pacific J. Math. (submitted).

10. M. Henriksen, Oh a class of regular rings that are elementary divisor rings, Arch. Math. 24 (1973), 133-141.

11. N. Jacobson, Lectures in abstract algebra, Vol. 2, Van Nostrand, Princeton, N.J., 1953, p. 46. MR 14, 837.

12. C. D. Meyer, Jr., Generalized inverses of block triangular matrices, SIAM J. Appl. Math. 19 (1970), 741-750. MR 42 \#7676.

13. M. Rosenblum, The operator equation $B X-X A=Q$ with selfadjoint $A$ and $B$, Proc. Amer. Math. Soc. 20 (1969), 115-120. MR 38 \#1537.

14. W. E. Roth, The equations $A X-Y B=C$ and $A X-X B=C$ in matrices, Proc. Amer. Math. Soc. 3 (1952), 392-396. MR 13, 900.

15. I. Vidav, Modules over regular rings, Math. Balkan. 1 (1971), 287-292. MR 44 \#6753.

Department of Mathematics, North Carolina State University Raleigh, North CaroLINA 27607 